\title{
Evaluation of treatment efficacy of tyrosine kinase inhibitors in rare single EGFR Exon 21 L861Q mutation; single center experience.
}

\section{pınar Gürsoy ( $\nabla$ pinargursoy77@gmail.com )}

ege university

\section{Research Article}

Keywords: Non-small cell lung cancer, Exon 21, L861Q, Erlotinib, Afatinib

Posted Date: December 13th, 2021

DOI: https://doi.org/10.21203/rs.3.rs-1161782/v1

License: (9) This work is licensed under a Creative Commons Attribution 4.0 International License. Read Full License 


\section{Abstract}

Aim:

Epidermal growth factor receptor (EGFR) mutations are the second most common oncogenic driver event in non-small cell lung cancer (NSCLC). We aimed to compare the first generation erlotinib treatment with the second generation afatinib treatment in patients with Non-small Cell Lung Cancer (NSCLC) with Epidermal growth factor receptor (EGFR) Exon 21 L861Q mutation.

\section{Patients and Methods:}

Progression-free survival (PFS) and overall survival (OS) of 30 NSCLC patients treated with erlotinib or afatinib due to single EGFR L861Q positivity were compared retrospectively.

\section{Results:}

There were twenty-three patients in the erlotinib arm and seven patients in the afatinib arm. Median PFS was 12.8 months in the erlotinib group and 9.3 months in the afatinib group. Median overall survival in erlotinib and afatinib groups were 77.9 months and 30.3 months, respectively. No statistically significant difference was found in the comparison of these survival times.

\section{Conclusion:}

Survival times of erlotinib and afatinib treatment are similar in patients with a single EGFR L861Q mutation. In patients receiving tyrosine kinase inhibitors (TKI) treatment, female gender has a positive effect on PFS and being none-smoker has a positive effect on OS. In patients with rare mutation Exon 21 L861Q positivity both first-generation and second-generation TKIs should be considered.

\section{Main Points}

1. L861Q mutation is a rare EGFR mutatıon. While many studies have reported TKI response for complex mutations, the number of studies with single exon 21 L861Q mutation is very limited.

2. There is no study in the literature comparısonıng the first generatıon EGFR-TKI and the second generatıon EGFR-TKI with single exon 21 L861Q mutation.

3. Survival times of erlotinib and afatinib treatment are similar in patients with a single EGFR L861Q mutation

4. In patients receiving tyrosine kinase inhibitors (TKI) treatment, female gender has a positive effect on PFS and being none-smoker has a positive effect on OS.

\section{Introduction}


Non-small cell lung cancer (NSCLC) is the most common cause of cancer-related death worldwide ${ }^{1}$. Epidermal growth factor receptor (EGFR) mutation in NSCLC is the most common mutation after K-RAS. EGFR mutation is found in $10-15 \%$ of all NSCLC cases in Western European populations, while the rate of EGFR mutation is 30\% in East Asian populations" ${ }^{2}$. Exon 19 and exon 21 L858R are called "classic EGFR mutations" and account for $85 \%$ of EGFR mutations ${ }^{3}$. These classic EGFR mutations show high sensitivity to treatment with Tyrosine kinase inhibitors (TKI). Studies have shown that TKI therapy results in prolongations in progression-free survival (PFS) compared to cytotoxic chemotherapy in these patients ${ }^{4}$. Exon 18, exon 20 and exon $21 \mathrm{~L} 861 \mathrm{Q}$ mutations are rarer mutations, and their response to TKI and their prognosis are unclear ${ }^{5-6}$.

Erlotinib and gefitinib are reversible first-generation TKIs, and afatinib is irreversible second-generation $\mathrm{TKIs}^{7-8}$. In studies with classical EGFR mutations, both first-generation TKI and second-generation TKI responses were found to be similar. Post-hoc analyses of LUX-lung 2, LUX-lung 3 and LUX-lung 6 studies have shown that "rare mutations" such as Exon 18, exon 20 and exon 21 L861Q are more susceptible to second generation $\mathrm{TKI}^{9}$.

The Exon 21 L861Q mutation accounts for 3\% of EGFR mutations, and this mutation is usually found in "complex" with G719X and Exon 19 del. While many studies have reported TKI response for complex mutations, the number of studies with Single Exon $21 \mathrm{~L} 861 \mathrm{Q}$ mutation is very limited ${ }^{10}$.

The aim of our study is to determine the characteristics of the rare single exon 21 L861Q patient group and to show whether there is a difference between the survival times according to the TKI used.

\section{Patients And Methods}

\subsection{Patients:}

Patients who were followed up at Hospital between 2010 and 2020 and received TKI for single EGFR exon 21 L861Q positive metastatic NSCLC were included in the study. Patients with other mutations and complex mutations for EGFR were excluded from the study. EGFR mutation positivity in patients with NSCLC was determined by pyrosequencing method. Age, gender, smoking status, performance score, stages, metastasis sites, treatments received before and after, side effects and date of death of the patients were recorded. The general characteristics of these patients and the effectiveness of their treatment with TKI were evaluated retrospectively.

\subsection{Ethical Considerations}

This study was approved by Ethical Committy of (Decision number: (21-7T/4) All patients gave written informed consent.

\subsection{Statistical analysis:}


IBM SPSS Statistics Version 22 package program was used for statistical analysis. Whether the data was normally distributed was determined by Kolmogorov-Smirnov and Shapiro-Wilk tests. Mann-Whitney U test was used to compare continuous variables between groups, Chi-square test and Fisher's exact test were used to compare categorical variables. Kaplan-Meier test was used for survival analysis. Results are presented as, median (min-max) and number (percentage). A p value of $<0.05$ was considered statistically significant in all statistical analyzes.

\section{Results}

\subsection{Patient characteristics:}

Thirty patients who received TKI therapy for metastatic NSCLC single exon 21 L861Q mutation positivity were included in the study. The pathological type of all patients was adenocarcinoma. The median age was 70 (52.0-84.0), 14 patients (46.7\%) were female and 16 patients (53.3\%) were male. Demographic and clinical characteristics of the patients were presented in Table 1. Twenty-three (76.7\%) of the patients received first-generation $\mathrm{TKI}$, and 7 (23.3\%) of patients received second-generation TKI. TKIs were used in the first-line treatment in $15(50 \%)$ patients, in the second-line treatment in $11(36.7 \%)$ patients, and in the tertiary treatment in $4(13.3 \%)$ patients. The treatments received by the patients before and after TKI were shown in Table 2.

\subsection{PFS and OS:}

PFS was found to be 13.2 months $(95 \% \mathrm{Cl} 7.8-18.7)$ in all patient groups. The PFS was 12.8 months (95\% Cl7.2-18.4) in the erlotinib group, and 9.3 months $(95 \% \mathrm{Cl} 6.0-12.6)$ in the afatinib group, with no difference between the two groups $(p=0.751)$ (Figure 1$)$.

When the PFS was compared according to gender, the PFS of female patients was found to be longer than male patients (19.3 vs $7.3 p=0.005)$. Similarly, there was a statistically significant difference between the PFS of female patients and male patients in the Erlotinib group $(19.4$ months $(95 \% \mathrm{Cl} 8.9$ $29.8)$ vs. 7.1 months $(95 \% \mathrm{Cl} 4,7-9.5)$, respectively $p=0.008)$. Also, the PFS of female patients was longer in the afatinib group, but no statistical difference was found (10.4 months vs. 7.8 months $p=0.823$ ).

When the PFS was compared according to the smoking status of the patients, the PFS was found to be 13.8 months in the none-smokers group and 9 months in the ex-smokers group $(p=0.749)$. In the erlotinib group, patients who had never smoked had a longer PFS than patients who had smoked, although it was not statistically significant ( 14.6 months vs. 8.2 months $p=0.320$ ). In the afatinib group, the PFSs for these patients were found to be 10.9 months and 7.2 months, respectively $(p=0.264)$.

No better response was observed in any subgroup when PFSs were compared according to tumor stages, number of metastatic sites, and performance status (Table 3 ).

OS of all patients was 73 months $(95 \% \mathrm{Cl} 41.0-104)$. OS was 77.9 months $(95 \% \mathrm{Cl} 44.2-111.5)$ in the erlotinib group and 30.3 months $(95 \% \mathrm{Cl} 30.1-30.5)$ in the afatinib arm, and there was no statistically 
significant difference between the two groups $(p=0.265)$ (figure 2).

When OS was evaluated according to gender, the OS of male patients was found to be longer than female patients (76.1 vs $37.2 \mathrm{p}=0.649)$.

When OS was compared by smoking status, OS was statistically significantly longer in the none-smokers group ( 97.5 months $(95 \% \mathrm{Cl} 65.6-129.3)$ vs. 40 months $(95 \% \mathrm{Cl} 26.7-53.2$ respectively, $\mathrm{p}=0.026)$. Similar results were also observed in the Erlotinib group ( 97.5 months vs. 45.1 months, respectively, $p=0.083$ ).

In this study, 5 patients (16.7\%) had brain metastases. Patients with brain metastases had a PFS of 12.0 months, and patients without brain metastases had a PFS of 14.6 months $(p=0.862)$. All of these patients received erlotinib treatment, and the PFS of this group was calculated as 12.0 months $(95 \% \mathrm{Cl} 4-19.9)$. The OS of the patients with brain metastases was 40.9 months (95\% $\mathrm{Cl} 20.2-61.6)$, and the OS of the patients without brain metastases was found to be statistically significantly longer (40.9 months vs. 89.1, respectively, $p=0.045$ ).

Bone metastases are the most common site of metastasis, and there was no difference between the groups with and without bone metastases in terms of both PFS and OS ( $p: 0.407$ vs $p=0.558$ ).

There was no statistically significant difference between the PFS of the patients who received TKI in first line and those who received TKI in other steps (17.8 months vs 8.2 months $p=0.082)$. However, when the OS was compared between the groups, a statistically significant difference was observed (39.4 months vs. 97.5 months, respectively, $p=0.019$ ).

\subsection{Adverse effects:}

The most common side effects associated with erlotinib were rash (82.6\%) and diarrhea (56.5\%). In the afatinib group, rash was the most common side effect with a rate of $28.5 \%$. Paronychia, which is frequently observed in TKI treatment, was observed in 3 patients (13\%), while all patients were in the erlotinib group. When all side effects were evaluated, grade 3-4 side effects were presented with anemia in only 1 patient (4.3\%). Interstitial lung disease, especially with a poor prognosis, was seen only in 1 patient (4.3\%) who received erlotinib, and treatment-related death was not observed in either group. The side effects observed in patients due to TKI treatment were shown in Table 4.

When the relationship between rash and PFS and OS was evaluated, there was a statistically significant difference between the PFS of the patients with and without rash ( 10.6 months vs. 12.9 months, $p=0.030$, respectively), but there was no difference between the $O S(p=0.863)$.

\section{Discussion}

In studies with classical mutations (EGFR exon 19 and exon 21 L858R), a progression-free survival benefit was observed in patients receiving TKI compared with chemotherapy. There are no prospective studies evaluating the efficacy of TKI in rare mutations in the literature. Results evaluating the efficacy of 
TKI in these patients are generally obtained from post-hoc analyzes of other studies or case series. Exon $21 \mathrm{~L} 861 \mathrm{Q}$ mutations may be a single mutation or part of complex mutations. Our study is the first in the literature to compare the efficacy of erlotinib and afatinib in a single EGFR exon 21 L861Q mutation positive patient group.

There are analyses showing that EGFR phosphorylation is preserved in cells expressing L861Q following treatment with first-generation TKI, such as erlotinib, while EGFR phosphorylation is lost after treatment with afatinib. These data suggest that second-generation TKIs may be more effective in targeting L861Q mutations. Moreover, preclinical studies have shown that L861Q mutations are more resistant to first generation TKIs than L858R mutations, and more sensitive to afatinib and osimertinib treatment ${ }^{11}$. The reason for this sensitivity has been shown to be the irreversible covalent binding of these two drugs to the cysteine-797 residue in the ATP pocket ${ }^{12-13}$.

In the post-hoc analysis of LUX-lung 2, LUX-lung 3 and LUX-lung 6 studies, single L861Q positivity was detected in $16(16.0 \%)$ of 100 patients with positive rare mutation ${ }^{9}$. With afatinib treatment, ORR was $56.3 \%$, PFS was 8.2 months, and OS was 17.1 months in these patients. Yang et al. reported that 8.2 months of PFS and 17.1 months of OS were observed in patients receiving afatinib treatment in their series of 12 cases $^{14}$.

In the literature by Chiu et al. and Xu et al., in studies with 57 and 15 patients, erlotinib/gefitinib was used in single L861Q positive patients, and PFS was 8.1 months and OS was 22.0 months $^{15}$. In our study, OS and PFS were found to be longer in both erlotinib and afatinib groups compared to literature results.

The OPTIMAL study is a phase III study comparing erlotinib and chemotherapy in classical EGFR mutation-positive patients. In this study, PFS was 13.1 months and OS was 22.8 months in the erlotinib arm. In our study, while the PFS of the erlotinib arm was the same as in the literature, OS was longer than the literature. The longer OS may be due to the higher proportion of patients receiving chemotherapy in the next steps.

In the OPTIMAL study, the Exon 21 L858R classical mutation was more common in female and nonsmokers $^{16}$. In our study, the incidence of L861Q mutation was more common in the non-smoker group, but no difference was found in terms of gender.

Studies on the pharmacokinetics of TKls have shown that metabolic clearance of TKIs is increased in smokers compared to never-smokers. As a result, patients who none-smokers provided $36 \%$ more benefits ${ }^{17}$. In our study, PFS and OS were found to be longer in both the erlotinib and afatinib arms in none-smokers, and only the OS benefit was found to be statistically significant.

Whether the development of rash is an indicator of TKI effectiveness has been investigated in many studies. Although this predictive effect was found in most of the studies, this relationship could not be clearly demonstrated in a few studies ${ }^{18}$. In our study, unlike the literature, patients who did not develop rash had longer PFS and no difference was found in terms of OS. 
The main limitations of our study are the small number of patients and its retrospective nature. Since afatinib treatment receives late repayment in our country, there is a difference in the number of patients between the groups. The TKIs chosen for treatment are the physician's choice and may affect the results. However, our study has important findings as it is the first study to compare the efficacy of erlotinib and afatinib in a single EGFR exon 21 L861Q mutation positive patient group.

In conclusion, both PFS and OS were found to be similar with erlotinib and afatinib treatments in rare EGFR exon 21 L861Q mutation positive patients. In this patient group, female gender should be considered in terms of PFS benefit and being none-smoker should be considered in terms of OS benefit. However, randomized studies with larger numbers of patients are needed for clearer results.

\section{Declarations}

\section{Disclosure statement}

The authors declare no conflict of interest with regard to the present study.

\section{Ethical approval}

This study was approved by Ethical Committee( 21-7T/4). All patients gave written informed consent.

\section{References}

1. Siegel RL, Miller KD, Jemal A (2019) Cancer statistics. CA Cancer J Clin 69:7-34.

2. D’Angelo S, Pietanza M.C, Johnson M.L, J Riely G, Miller V, S Sima C, et al. Incidence of EGFR exon 19 deletions and L858R in tumor specimens from men and cigarette smokers with lung adenocarcinomas, J. Clin.Oncol. 29 (15) (2011) 2066-2070.

3. Dogan S, Shen R, Ang D.C, Johnson M.L, D'Angelo S, Paik P.K, et al. Molecular epidemiology of EGFR and KRAS mutations in 3,026 lung adenocarcinomas: higher susceptibility of women to smoking-related KRAS-mutant cancers. Clin Cancer Res. 2012 Nov 15;18(22):6169-77.

4. Gazdar A.F. Activating and resistance mutations of EGFR in non-small-cell lung cancer: role in clinical response to EGFR tyrosine kinase inhibitors. Oncogene. 2009 Aug;28 Suppl 1.24-31.

5. Pao W. Chmielecki J. Rational, biologically based treatment of EGFR-mutant non-small-cell lung cancer. Nat Rev Cancer 10, 760-774.

6. Mitsudomi T, Kosaka T, Yatabe Y. Biological and clinical implications of EGFR mutations in lung cancer. Int J Clin Oncol 2006 Jun;11(3):190-8.

7. Zhou C, Wu YL, Chen G, Feng J, Liu X-Q, Wang C,et al. Erlotinib versus chemotherapy as first-line treatment for patients with advanced EGFR mutation-positive non-small-cell lung cancer (OPTIMAL, 
CTONG-0802): a multicentre, open-label, randomised, phase 3 study. The Lancet Oncology. 2011; $12: 735-742$.

8. Sequist L.V, Yang JC, Yamamoto N, O'Byrne K, Hirsh V, Mok T, et al. Phase III study of afatinib or cisplatin plus pemetrexed in patients with metastatic lung adenocarcinoma with EGFR mutations. Journal of clinical oncology. 2013; 31:3327-3334.

9.Yang JC, Sequist L.V, Geater S.L, Tsai C-M, Mok T, Schuler M, et al. Clinical activity of afatinib in patients with advanced nonsmall-cell lung cancer harbouring uncommon EGFR mutations: a combined posthoc analysis of LUX-Lung 2, LUX-Lung 3, and LUX-Lung 6, Lancet Oncol. 16 (2015) 830-838.

10. Chiu CH, Yang C, Shih J, Huang M-S, SuW-C, Lai R-S, et al. Epidermal Growth Factor Receptor Tyrosine Kinase Inhibitor Treatment Response in Advanced Lung Adenocarcinomas with G719X/L861Q/S768I Mutations. J Thorac Oncol 2015;10:793-9.

11. Banno E, Togashi Y, NakamuraY, Chiba M, Kobayashi $Y$, Hayashi $H$, et al. Sensitivities to various epidermal growth factor receptor-tyrosine kinase inhibitors of uncommon epidermal growth factor receptor mutations L861Q and S768I: What is the optimal epidermal growth factor receptor-tyrosine kinase inhibitor? Cancer Sci. 2016 Aug;107(8):1134-40.

12. Zhou W, Ercan D, Chen L, Yun C-H, Li D, Capelletti M, et al. Novel mutant-selective EGFR kinase inhibitors against EGFR T790M. Nature 2009; 462: 1070-4.

13. Walter AO, Sjin RT, Haringsma HJ, Ohashi K, Sun J, Lee K,et al .Discovery of a mutant-selective covalent inhibitor of EGFR that overcomes T790M-mediated resistance in NSCLC. Cancer Discov 2013; 3 : 1404-15.

14. Cho J.H, Lim S.H, An H.J, Kim K.H, Park K.U, Kang E.U,et al. An open-label, multicenter, phase II single arm trial of osimertinib in non-small cell lung cancer patients with uncommon EGFR mutation (KCSG-LU15-09), J. Clin. Oncol. 36 (15_suppl) (2018) 9050.

15. Rosell R, Ichinose $Y$, Taron M, Sarries C, Queralt C, Mendez P, et al . Mutations in the tyrosine kinase domain of the EGFR gene associated with gefitinib response in non-small-cell lung cancer. Lung Cancer 2005;50:25-33.

16. Rosell R ,Carcereny E , Gervais R, Vergnenegre A, Massuti B, Felip E, et al . Erlotinib versus standard chemotherapy as first-line treatment for European patients with advanced EGFR mutation-positive nonsmall-cell lung cancer (EURTAC): a multicentre, open-label, randomised phase 3 trial. Lancet Oncol 2012;13:239-46.

17. Hamilton M.Wolf J.L, Rusk J, Beard S-E, Clark G-M, Witt K,et al. Effects of smoking on the pharmacokinetics of erlotinib.Clin Cancer Res. 2006; 12: 2166-2171. 
18. Kainis I, Syrigos N, Kopitopoulou A, Gkiozos I, Filiou E, Nikolaou V,et al. Erlotinib-Associated Rash in Advanced Non-Small Cell Lung Cancer: Relation to Clinicopathological Characteristics, Treatment Response, and Survival. Oncol Res. 2018; 26(1): 59-69.

\section{Tables}

Table 1: Demographic data and clinical characteristics 


\begin{tabular}{|c|c|}
\hline Patients characteristics & $\mathrm{n}(\%)$ \\
\hline Age (mean) (min-max) & $70.0(52.0-84.0)$ \\
\hline \multicolumn{2}{|l|}{ Sex n(\%) } \\
\hline Female & $14(46.7 \%)$ \\
\hline Male & $16(53.3 \%)$ \\
\hline \multicolumn{2}{|l|}{ Smoking status n(\%) } \\
\hline None-smoker & $16(53.3 \%)$ \\
\hline Ex-smoker & $14(46.7 \%)$ \\
\hline Smoking package*year (min-max) & $50.0(30.0-60.0)$ \\
\hline \multicolumn{2}{|l|}{ ECOG performance status (\%) } \\
\hline 0 & $3(10 \%)$ \\
\hline 1 & $18(60 \%)$ \\
\hline 2 & $9(30 \%)$ \\
\hline 3 & $0(0 \%)$ \\
\hline \multicolumn{2}{|l|}{ Stage $n(\%)$} \\
\hline I & - \\
\hline II & $4(13.3 \%)$ \\
\hline III & - \\
\hline IV & $26(86.7 \%)$ \\
\hline \multicolumn{2}{|l|}{ Metastasis n(\%) } \\
\hline Solitary & $12(40.0 \%)$ \\
\hline Multiple & $18(60.0 \%)$ \\
\hline Brain metastases n(\%) & $5(16.7 \%)$ \\
\hline Bone metastases n(\%) & $12(40.0 \%)$ \\
\hline \multicolumn{2}{|l|}{ TKI type $\mathrm{n}(\%)$} \\
\hline Erlotinib & $23(76.7 \%)$ \\
\hline Afatinib & $7(23.3 \%)$ \\
\hline \multicolumn{2}{|l|}{ TKI treatment $\mathrm{n}(\%)$} \\
\hline First line & $15(50.0 \%)$ \\
\hline Second line & $11(36.7 \%)$ \\
\hline
\end{tabular}

Page 10/14 
Table 2: The treatments received by the patients before and after tyrosine kinase inhibitors.

\begin{tabular}{|llll|}
\hline Type of treatment received & First line $(\mathrm{n})$ & Second line $(\mathrm{n})$ & Third line $(\mathrm{n})$ \\
\hline Paclitaxel-platinum & 1 & 2 & 0 \\
\hline Docetaxel-platinum & 5 & 0 & 5 \\
\hline Pemetrexed -platinum & 6 & 3 & 0 \\
\hline Gemcitabine-platinum & 0 & 4 & 0 \\
\hline Vinorelbine -platinum & 3 & 0 & 0 \\
\hline Others & 0 & 0 & 0 \\
\hline
\end{tabular}

Table 3: Comparison of PFS and OS according to demographic and clinical characteristics 


\begin{tabular}{|c|c|c|c|c|}
\hline & \multicolumn{2}{|l|}{ PFS } & \multicolumn{2}{|l|}{ os } \\
\hline & Median months $(95 \% \mathrm{Cl})$ & $P$ value & Median months $(95 \% \mathrm{Cl})$ & $P$ value \\
\hline Overall & $13.2(7.8-18.7)$ & & $73.0(41.9-104.1)$ & \\
\hline \multicolumn{5}{|l|}{ Gender } \\
\hline Male & $7.3(5.3-9.3)$ & 0.005 & $76.1(42.2-110)$ & 0.649 \\
\hline Female & $19.3(9.8-29.2)$ & & $37.2(33.2-41.2)$ & \\
\hline \multicolumn{5}{|l|}{ Smoking status } \\
\hline None-smoker & $13.8(6.9-20.8)$ & 0.749 & $97.5(65.6-29.3)$ & 0.026 \\
\hline Ex-smoker & $9.0(6.4-11.6)$ & & $40.0(26.7-53.2)$ & \\
\hline \multicolumn{5}{|l|}{ Stage } \\
\hline 1 & - & 0.282 & - & 0.762 \\
\hline II & $12.7(12.0-13.3)$ & & $36.3(27.8-44.9)$ & \\
\hline III & - & & - & \\
\hline IV & $12.7(6.9-18.5)$ & & $77.9(44.2-111.5)$ & \\
\hline \multicolumn{5}{|l|}{ ECOG } \\
\hline 0 & $6.0(5.3-6.7)$ & 0.594 & - & 0.160 \\
\hline 1 & $11.6(8.8-14.5)$ & & 86.3 (52.6-119.9) & \\
\hline 2 & $19.9(8.8-30.1)$ & & $40.9(20.2-61.6)$ & \\
\hline 3 & - & & - & \\
\hline \multicolumn{5}{|l|}{ Metastasis type } \\
\hline Solitary & $8.3(5.7-10.9)$ & 0.341 & $74.7(40.2-109)$ & 0.448 \\
\hline Multiple & $14.9(7.7-22.1)$ & & $37.6(34.4-40.9)$ & \\
\hline
\end{tabular}

Table 4: Evaluation of side effects according to tyrosine kinase type 


\begin{tabular}{|lllll|}
\hline & Erlotinib & & Afatinib \\
\hline & Grade 1/2 n (\%) & Grade 3/4 n (\%) & Grade 1/2 n (\%) & Grade 3/4 n (\%) \\
\hline Diarrhea & $13(56.5 \%)$ & 0 & $2(28.5 \%)$ & 0 \\
\hline Rash & $18(78.2 \%)$ & $1(4.3 \%)$ & $2(28.5 \%)$ & 0 \\
\hline Mucositis & $11(47.8 \%)$ & 0 & $2(28.5 \%)$ & 0 \\
\hline ALT/AST elevation & & & 0 & 0 \\
\hline Paronychia & $1(4.3 \%)$ & 0 & 0 & 0 \\
\hline Anemia & $3(13.0 \%)$ & 0 & $2(28.5 \%)$ & 0 \\
\hline Interstitial lung disease & $1(4.3 \%)$ & $1(4.3 \%)$ & 0 & 0 \\
\hline
\end{tabular}

Figures

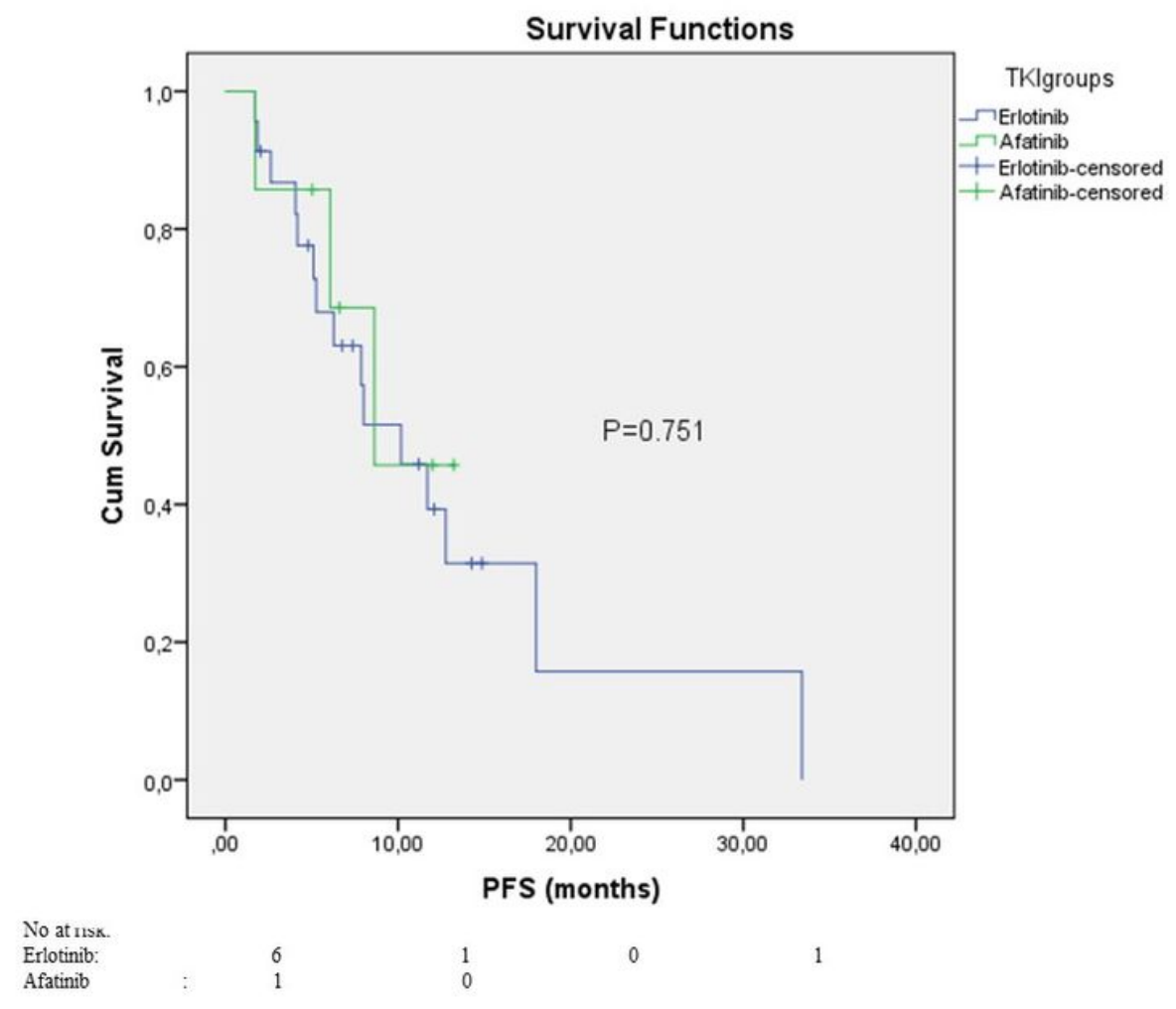

Figure 1 
Evaluation of PFS according to tyrosine kinase inhibitor types.

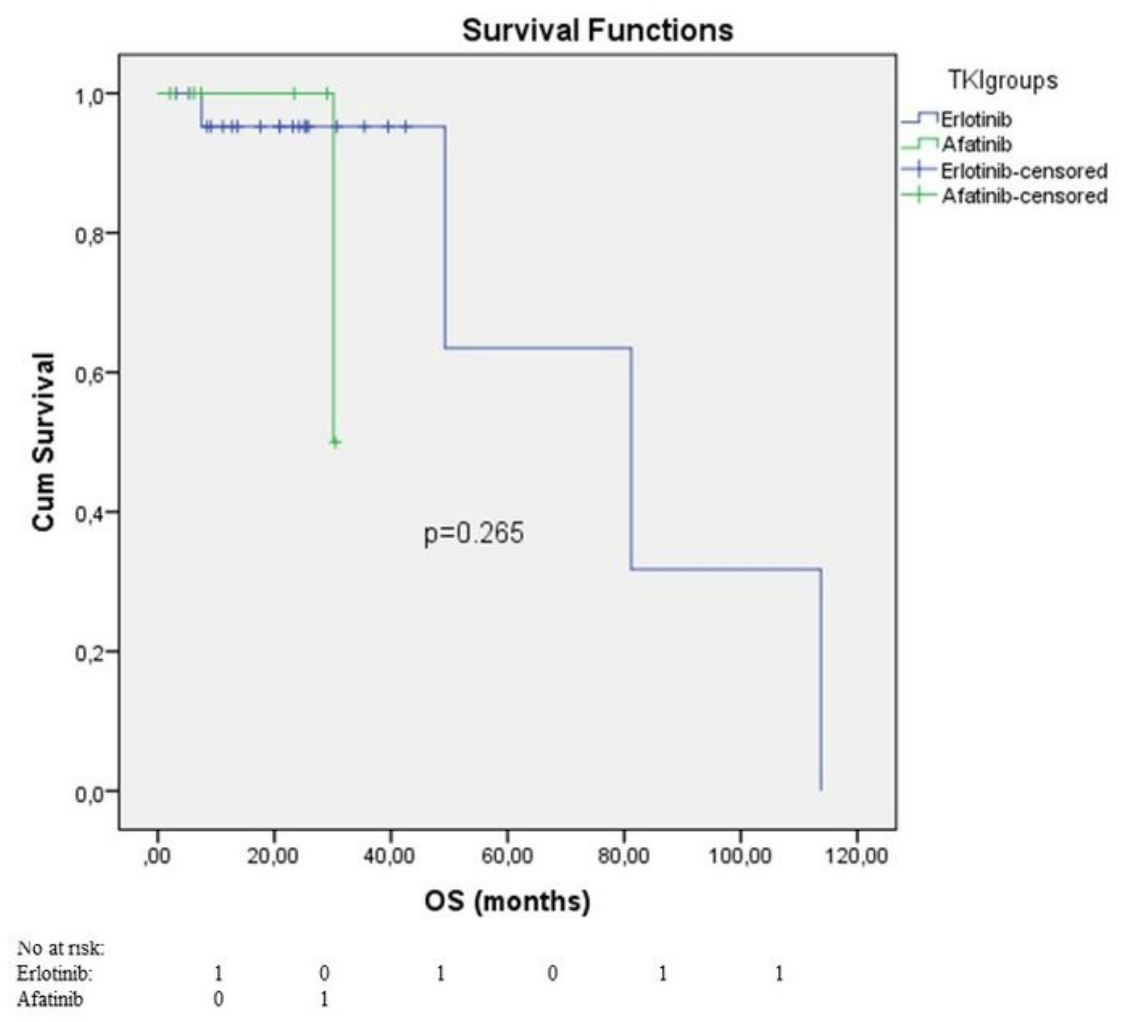

Figure 2

Evaluation of OS according to tyrosine kinase inhibitor types. 